\title{
A Bayesian Inference of Non-Life Insurance Based on Claim Counting Process with Periodic Claim Intensity
}

\author{
Uraiwan Jaroengeratikun, Winai Bodhisuwan, Ampai Thongteeraparp \\ Department of Statistics, Kasetsart University, Bangkok, Thailand \\ Email: ur_jaroen@yahoo.com, \{fsciwnb, fsciamu\}@ku.ac.th
}

Received January 22, 2012; revised February 25, 2012; accepted March 10, 2012

\begin{abstract}
The aim of this study is to propose an estimation approach to non-life insurance claim counts related to the insurance claim counting process, including the non-homogeneous Poisson process (NHPP) with a bell-shaped intensity and a beta-shaped intensity. The estimating function, such as the zero mean martingale (ZMM), is used as a procedure for parameter estimation of the insurance claim counting process, and the parameters of model claim intensity are estimated by the Bayesian method. Then, $\Lambda(t)$, the compensator of $N(t)$ is proposed for the number of claims in a time interval $(0, t]$. Given the process over the time interval $(0, t]$, the situations are presented through a simulation study and some examples of these situations are also depicted by a sample path relating $N(t)$ to its compensator $\Lambda(t)$.
\end{abstract}

Keywords: Estimating Function; Zero Mean Martingale; Non-Life Insurance Claim Counting Process; Non-Homogeneous Poisson Process; Bell-Shaped Intensity; Beta-Shaped Intensity

\section{Introduction}

In the field of non-life insurance, the modeling of claim counts is a very important component in a risk model with regard to loss reserving, pricing, underwriting, etc. The precision of a claim count estimation is the key to running an insurance business successfully. Jewell [1] presented the Bayesian credibility model, called the exact credibility model, for claim counts involving Bayesian analysis with a natural conjugate prior distribution. The exact credibility model for claim counts has been used in the non-life insurance industry as part of the process of estimating and predicting the expected claim counts in upcoming periods, using past experience of claims of a risk class or related risk classes, i.e., Jewell estimated $\mu\left(\Lambda_{j}\right)=E\left(N_{j(t+1)} \mid \Lambda_{j}\right)$, where $N_{j(t+1)}$ is a random variable representing the claim counts of the $j^{\text {th }}$ insurance contract during the $(t+1)^{\text {th }}$ period with the parameter $\Lambda_{j}$. The $N_{j}$ is independent and Poisson distributed as an exponential family with mean $E\left(N_{j s} \mid \Lambda_{j}\right)$, and variance $\operatorname{Var}\left(N_{j s} \mid \Lambda_{j}\right)$. The prior distribution of $\Lambda_{j}$ has a Gamma distribution with shape parameter $\gamma$ and scale parameter $\beta$. Also, the risk exposure or the number of policies of $j^{\text {th }}$ contract in each period $s$ is 1 , where

$j=1,2,3, \cdots, k$ and $s=1,2,3, \cdots, t$. The

$\hat{E}\left(\mu\left(\Lambda_{j}\right) \mid N_{j 1}, \cdots, N_{j t}\right)=\hat{E}\left(N_{j(t+1)} \mid N_{j 1}, \cdots, N_{j t}\right)$ is the estimator, i.e.
$\hat{E}\left(N_{j(t+1)} \mid N_{j 1}, \cdots, N_{j t}\right)=\hat{\mu}\left(\Lambda_{j}\right)=(1-z) \mu+z \bar{N}_{j} \quad$ where $\mu=E\left(\Lambda_{j}\right)=\frac{\gamma}{\beta}, z=\frac{t}{\beta+t}$ is the credibility factor, and $\bar{N}_{j}$ is the sample mean of $N_{j}$. Jewell's Bayesian credibility model was extended to others, such as the exact credibility model of Kaas, Dannenburg and Goovaerts (see [2]) and Ohlsson and Johansson (see [3]). Calculating the expected claim counts with the credibility approach only depends on the information from past experience of claim counts, and does not consider the occurrence behavior of claim counts over time. Some authors have considered the claim counts relating to a specified time or their behavior over time. Mikosch [4] viewed the claim counting process as a homogeneous Poisson process (HPP) in the Cramér-Lundberg model, one of the most popular and useful risk models in nonlife insurance. In non-life insurance portfolios, the claim counts during a time period are caused by periodic phenomena or seasonality. These claim counts are modeled in terms of a non-Homogeneous Poisson process (NHPP) with a period time-dependent intensity rate. Morales [5] studied the periodic risk model consisting of the claim counting process with a bell-shaped intensity function (called the Gaussian intensity) of the form

$$
\lambda(t)=\frac{\lambda^{*} \exp \left\{-\frac{1}{2 \sigma^{2}}\left(t-\frac{1}{2}\right)^{2}\right\}}{\left(\Phi\left(\frac{1}{2 \sigma}\right)-\Phi\left(-\frac{1}{2 \sigma}\right)\right) \sigma \sqrt{2 \pi}},
$$


for $\lambda(s+t)=\lambda(t), \quad t \in[0,1), \quad s=0,1,2, \cdots, \quad \lambda^{*}>0$ and $\sigma>0$, where $\lambda^{*}, \sigma$ and $s$ are the parameters of model periodic claim intensity and $\Phi$ is the standard normal distribution function. The unknown parameters of model intensity were estimated by the maximum likelihood estimation (MLE), and the ruin probability model was evaluated through a simulation study. Furthermore, $\mathrm{Lu}$ and Garrido [6] and Garrido and $\mathrm{Lu}$ [7] explored the periodic NHPP model with a beta-shaped intensity function,

$$
\lambda(t)=\frac{\lambda^{*}\left(\frac{t-[t]-m_{1}}{D}\right)^{p-1}\left(1-\frac{t-[t]-m_{1}}{D}\right)^{q-1}}{\alpha^{*}},
$$

for $D=m_{2}-m_{1}, \quad \alpha^{*}=\left(\frac{t^{*}-m_{1}}{D}\right)^{p-1}\left(1-\frac{t^{*}-m_{1}}{D}\right)^{q-1}$ is the scale factor, while $t^{*}=m_{1}+\frac{D(p-1)}{p+q-2}$ is the mode of function, $\lambda^{*}>0, \quad p, q \geq 1$ and $0 \leq m_{1}<m_{2} \leq 1$, where $\lambda^{*}, p$ and $q$ are the parameters, [.] is the greatest integer function, $m_{1}$ and $m_{2}$ represent the starting and ending point of the occurrence interval, respectively.

In this study, we present an estimation approach to non-life insurance claim counts in the claim counting processes using an estimating function, the zero mean martingale (ZMM). This approach provides a parameter estimator, $\hat{\Lambda}(t)$, of process, including the MLE for the parameter estimation of model claim intensity proposed by Jaroengeratikun et al. [8]. The $\hat{\Lambda}(t)$ can be interpreted as the insurance claim counts, $N(t)$, during the time interval $(0, t]$. The estimate $\hat{\Lambda}(t)$ is also useful for predicting time of claim occurrences or the claim counts in the next periods. In this paper, we extend their approach to estimate clam counts in an NHPP with periodic bell-shaped and beta-shaped intensities. Then, the Bayesian analysis is used to estimate the parameters of model claim intensity.

\section{Non-Life Insurance Claim Counting Process}

We define $N(t)=\#\left\{i \geq 1: T_{i} \leq t\right\} ; t \geq 0$ the cumulative number of insurance claims that have occurred during time $(0, t]$, where $T_{n}=W_{1}+\cdots+W_{n} ; n \geq 1$ is a claim arrival time and $W_{i}$ is an identically independent distributed (iid) with Exponential whose parameter is $\lambda\left(w_{i}\right)$, called the claim intensity rate.

$N=\{N(t) ; t \geq 0\}$ is a claim counting process, $N(t)$

can be written as $N(t)=\int_{0}^{t} \mathrm{~d} N(u)$ where $N(t)$ is an increment of $N$ in a small fraction period. In this study, the insurance claim counts are NHPP with periodic claim intensity rate, i.e. bell-shaped intensity function and betashaped intensity function. The bell-shaped intensity as an initial season, $s=0$, is given in Equation (1) [5], where $\lambda^{*}$ is an average number of claims over a period and $\sigma$ is a variability of season. The mean value function of $N(t)$ has the following representation

$$
\Lambda(t)=[t] \lambda^{*}+\frac{\lambda^{*}\left\{\Phi\left(\frac{t-[t]-\frac{1}{2}}{\sigma}\right)-\Phi\left(-\frac{1}{2 \sigma}\right)\right\}}{\left(\Phi\left(\frac{1}{2 \sigma}\right)-\Phi\left(-\frac{1}{2 \sigma}\right)\right)}
$$

The beta-shaped intensity function is given in Equation (2) $[6,7]$, where $\lambda^{*}$ is the peak level for claim intensity, Its integrated intensity is

$$
\Lambda(t)=\frac{\lambda^{*} D B_{p}\left(\frac{t-[t]-m_{1}}{D}\right)^{p-1}\left(1-\frac{t-[t]-m_{1}}{D}\right)^{q-1}}{\alpha^{*}},
$$

where $B_{p}=[t] B(p, q)+B\left(p, q ; \frac{t-[t]-m_{1}}{D}\right)$,

$$
\begin{aligned}
& B(p, q)=\int_{0}^{1} v^{p-1}(1-v)^{q-1} \mathrm{~d} v \text { and } \\
& B(p, q ; t)=\int_{0}^{t} v^{p-1}(1-v)^{q-1} \mathrm{~d} v .
\end{aligned}
$$

Both the bell-shaped and the beta-shaped intensity are depicted in Figure 1. In Figure 1(a) the claim of occurrence in the tail of the period, i.e. left and right tail of period, changes slowly. While the beta-shaped intensity is shown in Figure 1(b), the claim of occurrence in the left and right tail of the period changes quickly.

On a probability space $\left(\Omega, F, P_{\Lambda}\right), N(t)$ is modeled by NHPP with a mean value function or parameter

$$
\Lambda(t)=\int_{0}^{t} \lambda(u) \mathrm{d} u=E(N(t)) . \text { As }
$$

$\{\lambda(t)=\alpha(t) \cdot k(t) ; t \geq 0\} ;$ is called the multiplicative intensity, where $\alpha(t)$ and $k(t)$ are defined as the claim intensity rate and the exposure risk, respectively. We consider $N$ as a non-decreasing right continuous step function 0 at time $t=0$ and jumps of size 1 , and

$$
P_{\Lambda}=p(N(t) ; \Lambda)=\operatorname{Pr}\{N(t)=n\}=\frac{\Lambda(t)^{n} \exp (-\Lambda(t))}{n !}
$$

for $n=0,1,2, \cdots$ and

$$
\operatorname{Pr}\{\mathrm{d} N(t)=1\}=\lambda(t) \mathrm{d} t=E\{\mathrm{~d} N(t)\}
$$




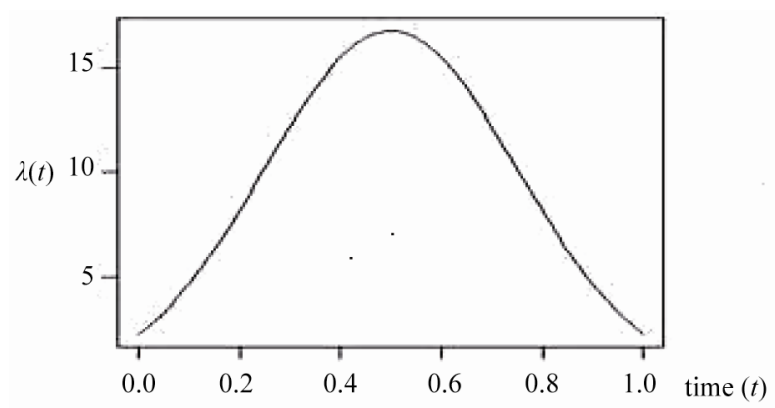

(a)

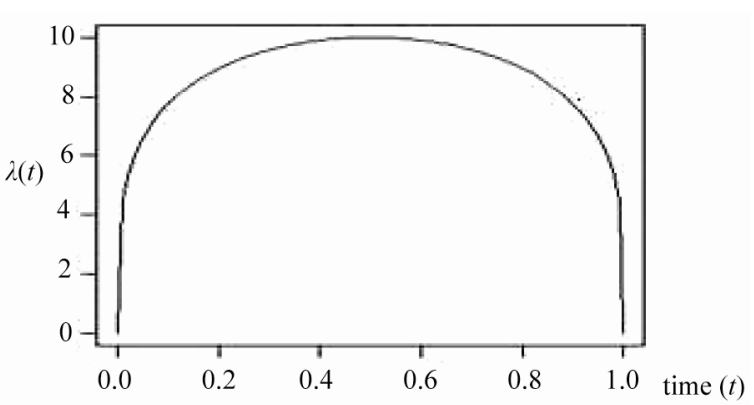

(b)

Figure 1. Non-life insurance claim intensity function, (a) Bell-shaped intensity $\lambda(t)=\frac{42}{\sqrt{2 \pi}} \exp \left\{-8\left(t-\frac{1}{2}\right)^{2}\right\}$ and (b) Betashaped intensity $\lambda(t)=10 \sqrt{2}(t-[t])^{1 / 4}(1-t+[t])^{1 / 4}$ while $p=q$.

\section{Parameter Estimation of the Non-Life Insurance Claim Counting Process}

In this section, we introduce the methods which are useful for parameter estimation of the non-life insurance claim counting process, including the estimating function, the martingale method, and the Bayesian estimation approach $(\mathrm{BE})$.

\subsection{Estimating Function}

On a probability space $\left(\Omega, F, P_{\Lambda}\right)$, where $\Lambda \in \Delta, \Delta$ is an open interval on the real line, $P_{\Lambda}=p(N(t) ; \Lambda)$. Suppose that the observation $N(t)=n$, the estimating functions, $g(N(t) ; \Lambda)$, are functions of $N(t)$ and the parameter $\Lambda$. By solving $g(N(t) ; \Lambda)=0$, a so-called estimating equation, an estimate of $\Lambda$ is obtained. Then $g(N(t) ; \Lambda)$ is an unbiased estimating function if $E[g(N(t) ; \Lambda)]=0$ for all $\Lambda \in \Delta$ (see [9]).

In this study, the estimating function for parameter estimation of the insurance claim counting process is provided by the martingale method.

\subsection{The Martingale Method}

The martingales are random processes relating to time. On a probability space $\left(\Omega, F, P_{\Lambda}\right)$, we suppose the increasing family $\wp=\left\{\wp_{t} ; t \geq 0\right\}$, a filtration or history $\wp_{t}$, which is the available data at the time $t$. The process $M=\{M(t) ; t \geq 0\}$ is a martingale with respect to $\wp$ if $E\{M(t)\}<\infty$ exist, and

$E\left\{M(t+s) \mid \wp_{t}\right\}=M(t)$ for all $s \geq 0$. As a result of the properties of the martingale, $E\{M(t)\}=E\{M(0)\}$ for all $t \geq 0$, then $E\{M(0)\}=0$ for a zero mean martingale $[10,11]$.

This study of the martingale method is useful for constructing an estimating function for a parameter estimation of the insurance claim counting process. The process takes place over a small time interval $(t, t+\mathrm{d} t]$, $E\left\{\mathrm{~d} N(t) \mid \wp_{t-}\right\}=\lambda(t) \mathrm{d} t$ and as a result of the meaning of martingale property, the martingale can be written as

$$
\mathrm{d} N(t)-\lambda(t) \mathrm{d} t=\mathrm{d} M(t)
$$

which is a martingale-difference. Then, the following martingale is

$$
\int_{0}^{t}\{\mathrm{~d} N(u)-\lambda(u) \mathrm{d} u\}=\int_{0}^{t} \mathrm{~d} M(u)
$$

or it can be rewritten in the form of $N(t)-\Lambda(t)=M(t)$, is a ZMM. Based on ZMM, we obtain $E\{M(t)\}=0=E\{N(t)-\Lambda(t)\}$.

Thus, $N(t)-\Lambda(t)=0$ is an estimating equation for parameter estimation of the insurance claim counting process. Also, as a result of the parameter estimate of the process, this can be interpreted as an $N(t)$ estimate or, in other words, $\Lambda(t)$ is called the compensator of $N(t)$, and this estimate is useful for predicting the times of occurrence of insurance claim counts [11]. We can depict the systematic part of the process of insurance claim counts, $N(t)$, related to its compensator, $\Lambda(t)$, and the associated martingale $N(t)-\Lambda(t)=M(t)$ in Figures 2(a) and (b), respectively, based on a sample of 15 independent random times of claims occurrence in the NHPP with a claim intensity $\lambda(t)$ where

$$
\lambda(t)=31 \exp \left\{-3\left(t-\frac{1}{2}\right)^{2}\right\}
$$

\subsection{A Bayesian Estimation Approach: The Model Specification for the Parameters of Model Claim Intensity}

In order to get the estimate of the compensator of $N(t)$, $\hat{\Lambda}(t)$, on the NHPP model of the non-life insurance claim counts, the parameters of the claim intensity func- 


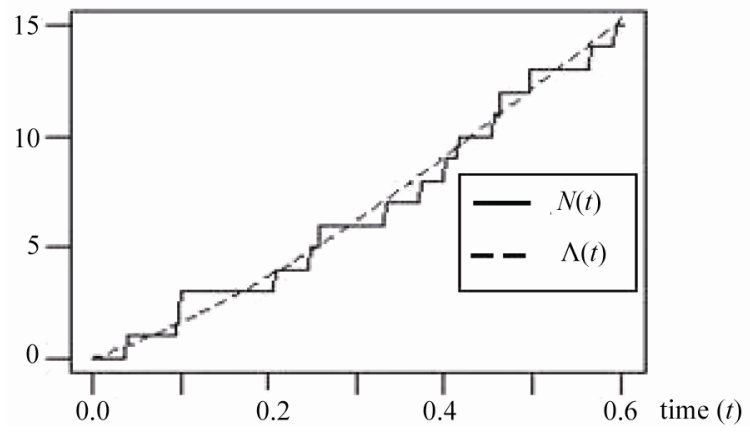

(a)

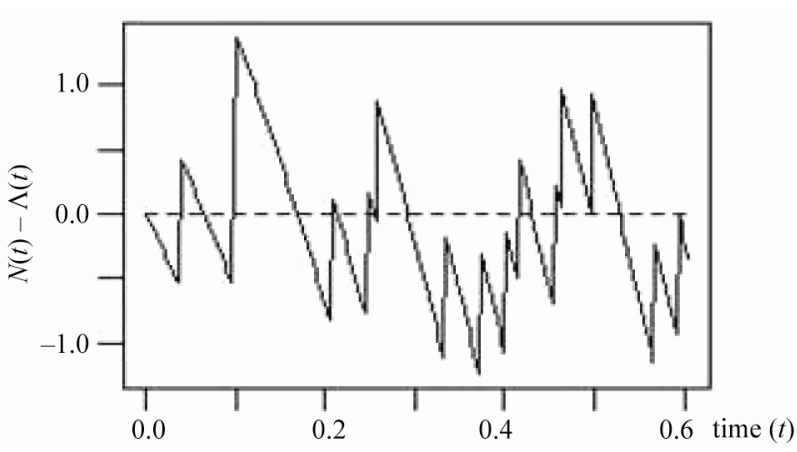

(b)

Figure 2. In a sample of 15 independent random times of claims occurrence with the claim intensity

$\lambda(t)=31 \exp \left\{-3\left(t-\frac{1}{2}\right)^{2}\right\}$, (a) Non-life insurance claim counting process $N(t)$ related to its compensator $\Lambda(t)$; and (b) Martingale $M(t)=N(t)-\Lambda(t)$.

tion are estimated by the BE. Given $N(t)=n$, we suppose that $t_{1}, t_{2}, t_{3}, \cdots, t_{N(t)}$ are the arrival times of the claims on the time interval $(0, t]$ with a cumulative distribution function (a general order statistics model)

$$
F(t)=1-\exp (-\Lambda(t)) \text {. }
$$

Its likelihood function $[4,12]$ is given by

$$
l(\boldsymbol{\theta} ; \xi)=\prod_{i=1}^{n} \lambda\left(t_{i}\right) \exp \left(-\Lambda\left(t_{n}\right)\right), \text { where } \boldsymbol{\theta} \text { is a vector of }
$$

the parameters of the model claim intensity, $\xi$ denotes the set of claim arrival times, the $\lambda\left(t_{i}\right)$ of both the bellshaped and the beta-shaped intensity function are given in Equations (1) and (2), respectively. While $\boldsymbol{\theta}=\left(\lambda^{*}, \sigma\right)^{\prime}$ of the bell-shaped intensity function and $\boldsymbol{\theta}=\left(\lambda^{*}, p, q\right)^{\prime}$ of the beta-shaped intensity function are unknown, the estimate of $\boldsymbol{\theta}$ can be obtained by a Bayesian analysis. The BE approach treats all unknown parameters in Equation (6) as random variables under their prior distribution and derives their conditional distribution upon the known information (sample data of claim arrival times). In this study, we apply the following prior density for

$$
\boldsymbol{\theta}=\left(\lambda^{*}, \sigma\right)^{\prime} \text { : }
$$

1) $\lambda^{*} \sim \operatorname{Gamma}\left(a_{1}, b_{1}\right)$,

2) $\sigma^{2} \sim \operatorname{Gamma}\left(a_{2}, b_{2}\right)$,

where $a_{1}, b_{1}, a_{2}$ and $b_{2}$ are known parameters, and the following prior density for $\boldsymbol{\theta}=\left(\lambda^{*}, p, q\right)^{\prime}$ :

1) $\lambda^{*} \sim \operatorname{Gamma}\left(c_{1}, d_{1}\right)$,

2) $p \sim \operatorname{Gamma}\left(c_{2}, d_{2}\right) \mathrm{I}(1$,$) ,$
3) $q \sim \operatorname{Gamma}\left(c_{3}, d_{3}\right) \mathrm{I}(1$,$) ,$

where $c_{1}, d_{1}, c_{2}, d_{2}, c_{3}$ and $d_{3}$ are known parameters and Gamma $(a, b)$ denotes a gamma distribution with mean $a / b$ and variance $a / b^{2}$. The restriction $p, q \geq 1$, is imposed with the construct $\mathrm{I}(1$,$) . We assume$ that among parameters, $\boldsymbol{\theta}$, are independent. The estimated parameters are based on the joint posterior density as,

$$
h(\boldsymbol{\theta} \mid \xi) \propto h(\boldsymbol{\theta}) l(\boldsymbol{\theta} ; \xi),
$$

where $h(\boldsymbol{\theta})$ is the joint prior density, and $l(\boldsymbol{\theta} ; \xi)$ is the likelihood function of $\xi$. The Equation (9) has a complicated form or no closed form. Therefore, the BE is implemented on the basis of the Markov chain Monte Carlo (MCMC) algorithms with the Gibbs sampler to solve these problems, see [13-16]. In this case, we estimate the parameters $\boldsymbol{\theta}$ with the empirical Bayes' estimator obtained by using the BUGS program.

\section{Simulation Study}

In this study, a simulation model is used to investigate how the observation of the non-life insurance claim counting process can be used to estimate its model parameter, i.e. claim intensity $\lambda(t)$ or in term $\Lambda(t)$, using the estimating function provided by the martingale method with ZMM. In particular, the NHPP of the insurance claim counts with bell-shaped and beta-shaped intensities, we consider the simulation study of the processes of the insurance claim counts during the claim time interval $(0, t]$ in which the observation involves the claim arrival times, $t_{1}, t_{2}, t_{3}, \cdots, t_{N(t)}$. The claim arrival times can be simulated by using the mean value function $\Lambda(t)$ as a claim arrival time of the HPP with mean one [5]. It implies that $E_{1}, E_{2}, E_{3}, \cdots, E_{n}$ are independent and exponentially distributed with mean one, where 
$E_{i}=\Lambda\left(t_{i}\right)-\Lambda\left(t_{i-1}\right)$, for all $i=1,2,3, \cdots, n$. So, the $n^{\text {th }}$ claim arrival time, $t_{N(t)=n}$, is generated by $[5,8]$

$$
t_{N(t)=n}=\Lambda^{-1}\left(E_{1}+E_{2}+E_{3}+\cdots+E_{n}\right),
$$

where $\Lambda^{-1}\left(E_{i}\right)$ is the invertible function of $\Lambda(t)$, with $\lambda^{*}=0.1, \sigma=0.25$ and $\lambda^{*}=10, \sigma=0.25$ for the model bell-shaped intensity, and $\lambda^{*}=0.1$, $p=q=1.25$ and $\lambda^{*}=10, p=q=1.25$ for the model beta-shaped intensity.

In this simulation study of the non-life insurance claim counting process over the time interval $(0, t]$, the number of observations, $N(t)=n$, is composed of $5,10,15$ and 20. The processes are carried out with 5000 sample paths. In each sample path, the parameter estimate of the model claim intensity is computed using the $\mathrm{BE}$ method with the prior distributions given in Equations (7) and (8) where $a_{1}=0.01, b_{1}=0.01, a_{2}=5, b_{2}=1, c_{1}=0.1$, $d_{1}=0.1, c_{2}=5, d_{2}=1, c_{3}=5, d_{3}=1$, and the estimating function, such as the ZMM which is used to estimate the parameter $\Lambda(t)$ of the process (or the compensator $\Lambda(t)$ of $N(t)$ ), i.e. fitting the compensator estimate $\hat{\Lambda}(t)$ to $N(t)$. Also, the mean squared error (MSE) is provided to measure the fitting $\hat{\Lambda}(t)$ to $N(t)$ as the following form

$$
\text { MSE }=\sum_{i=1}^{p} \frac{\int_{0}^{t}\left(\hat{\Lambda}_{i}(u)-N_{i}(u)\right)^{2} \mathrm{~d} u}{S_{p}},
$$

where $S_{p}$ denotes the number of sample paths. Notice that the MSE of the compensator estimate $\hat{\Lambda}(t)$ of $N(t)$ for the processes, as shown in Tables $\mathbf{1}$ and $\mathbf{2}$, depends on the parameters of the model claim intensity as in the following results, for the NHPP with a bellshaped intensity, the parameters of its model claim intensity $\lambda^{*}=0.1, \sigma=0.25$ (a small average number of claims over a period), the MSE of the compensator estimate $\hat{\Lambda}(t)$ of $N(t)$ increases as the observation number increases. In the same process with the parameters of model claim intensity $\lambda^{*}=10, \sigma=0.25$, the MSE of the compensator estimate $\hat{\Lambda}(t)$ of $N(t)$ decreases

Table 1. MSE of the compensator estimate $\hat{\Lambda}(t)$ of $N(t)$ in the NHPP of the non-life insurance claim counts with bell-shaped intensity.

\begin{tabular}{cccc}
\hline$\lambda^{*}$ & $\sigma$ & $N(t)$ & MSE \\
\hline \multirow{2}{*}{0.1} & 0.25 & 5 & 0.873166 \\
& & 10 & 1.663399 \\
& & 15 & 2.428750 \\
& & 20 & 3.141596 \\
10 & 0.25 & 5 & 5.968821 \\
& & 10 & 5.676725 \\
& & 15 & 4.630684 \\
& & 20 & 4.880201 \\
\hline
\end{tabular}

Table 2. MSE of the compensator estimate $\hat{\Lambda}(t)$ of $N(t)$ in the NHPP of the non-life insurance claim counts with beta-shaped intensity.

\begin{tabular}{cccc}
\hline$\lambda^{*}$ & $p=q$ & $N(t)$ & MSE \\
\hline \multirow{2}{*}{0.1} & 1.25 & 5 & 0.877067 \\
& & 10 & 1.883131 \\
& & 15 & 2.821956 \\
& & 20 & 4.170855 \\
10 & \multirow{2}{*}{1.25} & 5 & 0.885957 \\
& & 10 & 1.971573 \\
& & 15 & 4.469941 \\
& & 20 & 5.246322 \\
\hline
\end{tabular}

while its observation number increases until the observed 15 times of claims occurrence, and then its MSE values begin to increase as the observation number increases. For the NHPP with a beta-shaped intensity $\lambda^{*}=0.1$, $p=q=1.25$ (a small peak level for claim intensity) and $\lambda^{*}=10, p=q=1.25$, the MSE of the compensator estimate $\hat{\Lambda}(t)$ of $N(t)$ increases exponentially as their observation number increases.

Some examples in these situations of the NHPP with both bell-shaped and beta-shaped intensities, of non-life insurance claim counts based on a sample of 5, 10, 15 and 20 times of claims occurrence, are illustrated in Figures 3 and $\mathbf{4}$, including the $N(t)$ and its compensator $\Lambda(t)$. Figure 3 shows a sample path of the process with a bell-shaped intensity $\lambda^{*}=10, \sigma=0.25$. The $N(t)$ and its compensator $\Lambda(t)$ are characterized by the parameters of model claim intensity $\lambda^{*}=10, \sigma=0.25$, the compensator $\Lambda(t)$ fits well with $N(t)$, as the observation number is 15 and 20 (slightly larger than the claim intensity $\left.\lambda^{*}=10\right)$. While the $N(t)$ and its compensator $\Lambda(t)$ in the process with a beta-shaped intensity $\lambda^{*}=10, p=q=1.25$ is shown in Figure 4, the compensator $\Lambda(t)$ fits with $N(t)$, as the observation number is small.

\section{Conclusions and Discussion}

\subsection{Conclusions}

In a non-life insurance claim counting process over the time interval $(0, t]$, the simulation study of the NHPP with both bell-shaped and beta-shaped intensities demonstrates the fitting of the compensator estimate $\hat{\Lambda}(t)$ to $N(t)$. The model fitting depends on the parameters of model claim intensity and model specification of claim intensity. Firstly, regarding the NHPP with the parameters of the model bell-shaped intensity, a $\lambda^{*}$ has almost no claim occurrences over a period and any $\sigma$ The compensator estimate $\hat{\Lambda}(t)$ is a good fit to $N(t)$ with small MSE and small number of observations. In the same process with the parameters of the model claim intensity, an average number of claims over a period $\lambda^{*}$ 


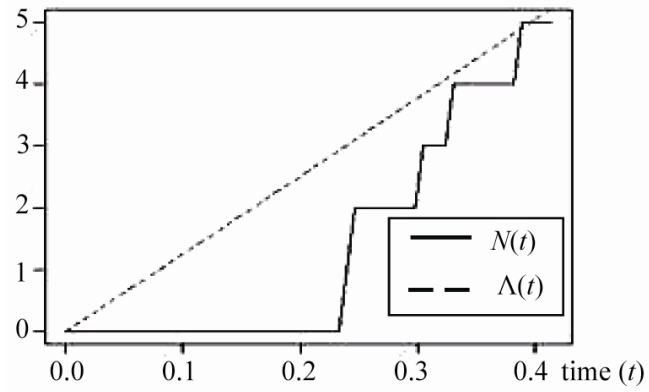

(a)

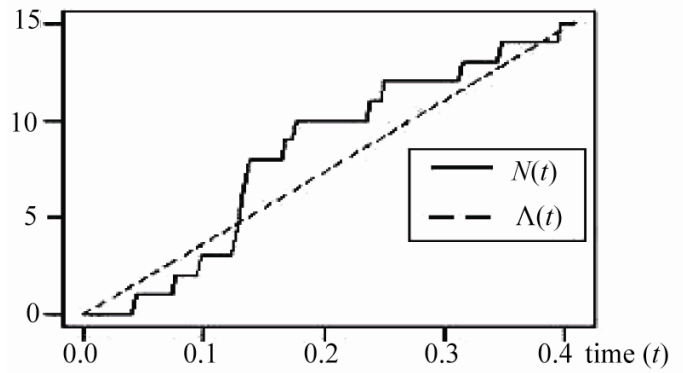

(c)

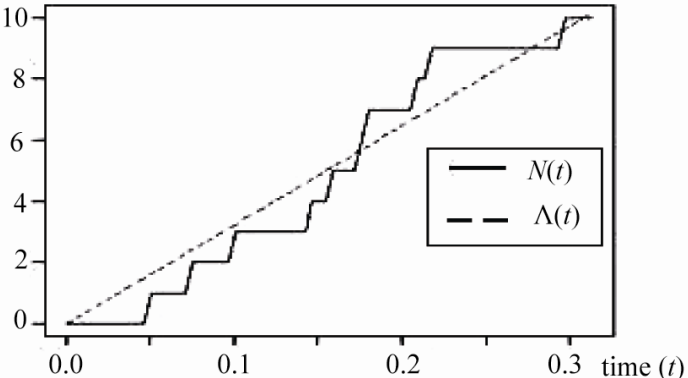

(b)

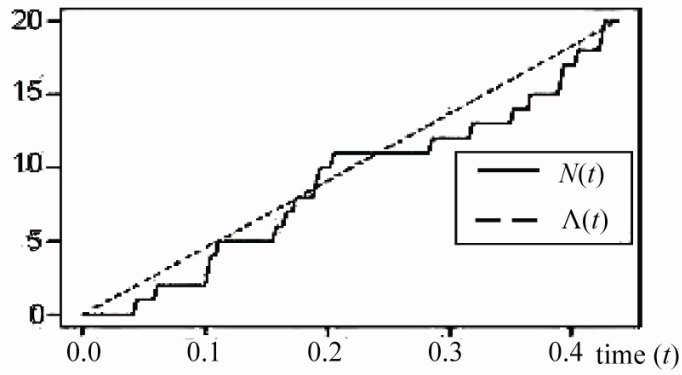

(d)

Figure 3. $N(t)$ and its compensator $\Lambda(t)$ in the NHPP with the parameters of a bell-shaped intensity $\lambda^{*}=10, \sigma=0.25$ based on a sample of (a) 5 claims; (b) 10 claims; (c) 15 claims; and (d) 20 claims.

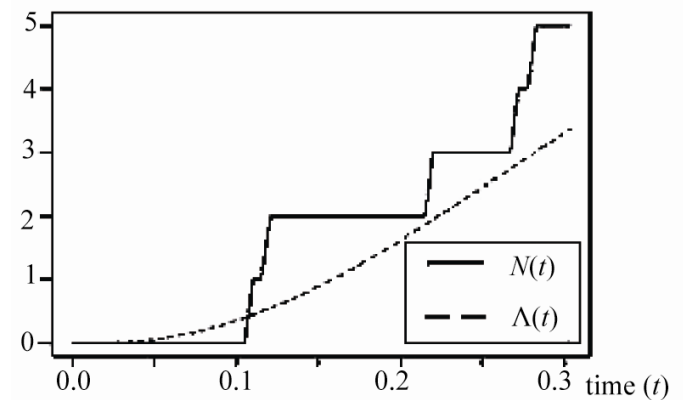

(a)

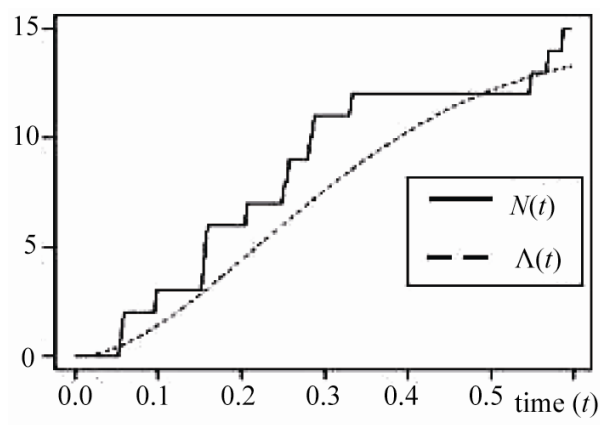

(c)

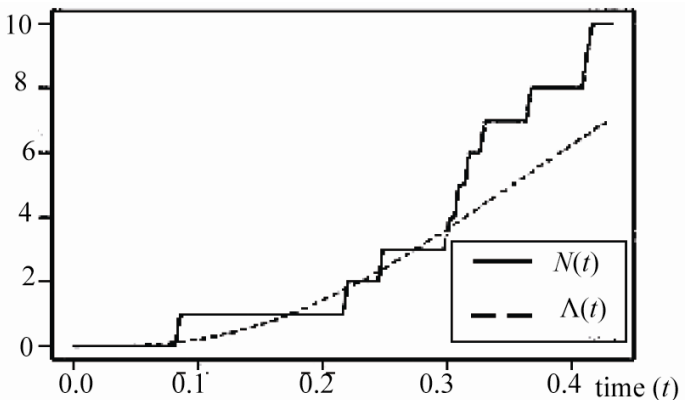

(b)

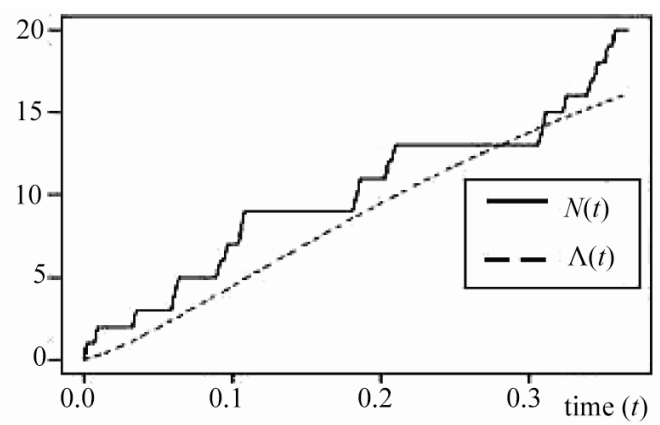

(d)

Figure 4. $N(t)$ and its compensator $\Lambda(t)$ in the NHPP with the parameters of a beta-shaped intensity $\lambda^{*}=10, p=q=1.25$ based on a sample of (a) 5 claims; (b) 10 claims; (c) 15 claims; and (d) 20 claims.

is not less than one and any $\sigma$. The MSE of the compensator estimate $\hat{\Lambda}(t)$ of $N(t)$ is small while the number of observations is slightly larger than the value of $\lambda^{*}$. Secondly, when the model beta-shaped intensity is considered with the parameters $p=q$, the compensator estimate $\hat{\Lambda}(t)$ is a good fit to $N(t)$ with small MSE and small number of observations. Some examples of the situations in the simulation study are also depicted 


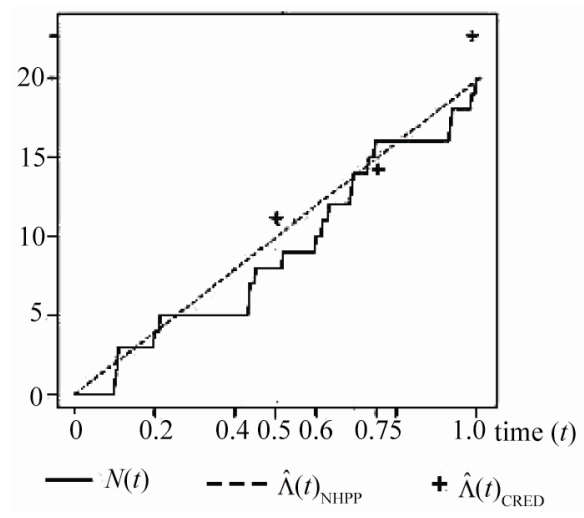

Figure 5. $N(t), \hat{\Lambda}(t)_{\mathrm{NHPP}}$, and $\hat{\Lambda}(t)_{\mathrm{CRED}}$ in the NHPP with a bell-shaped intensity of non-life insurance claim counts.

by a sample path relating $N(t)$ and its compensator $\Lambda(t)$.

\subsection{Discussion}

This study is a parameter estimation approach to non-life insurance claim counting process. The parameter $\Lambda(t)$ of the claim counting process is estimated from observations using an estimating function, such as ZMM. A result of the parameter estimate $\hat{\Lambda}(t)$ can be interpreted as an $N(t)$, called the compensator estimate $\hat{\Lambda}(t)$ of $N(t)$. This estimate is also useful for predicting the time of insurance claim occurrences. Using an example, we can depict a comparison of the two approaches of nonlife insurance claim counts, including this insurance claim counting process and the Jewell's credibility approach. In Figure 5, the compensator $\hat{\Lambda}(t)_{\mathrm{NHPP}}$ in the NHPP with a bell-shaped intensity (dashed line) is a good fit to $N(t)$ over time $(0, t]$; on the other hand, the procedure of the credibility estimate $\hat{\Lambda}(t)_{\mathrm{CRED}}(+)$ or the compensator $\hat{\Lambda}(t)_{\mathrm{CRED}}$ of $N(t)$ on the credibility model does not consider the behavior of insurance claim counts over time.

\section{REFERENCES}

[1] W. S. Jewell, "Credible Means Are Exact Bayesian for Exponential Families," ASTIN Bulletin, Vol. 8, No. 1, 1974, pp. 77-90.

[2] R. Kaas, D. Dannenburg and M. Goovaerts, "Exact Credibility for Weighted Observations," ASTIN Bulletin, Vol. 27, No. 2, 1997, pp. 287-295. doi:10.2143/AST.27.2.542053

[3] E. Ohlsson and B. Johansson, "Exact Credibility and
Tweedie Models," ASTIN Bulletin, Vol. 36, No. 1, 2006 , pp. 121-133. doi:10.2143/AST.36.1.2014146

[4] T. Mikosch, "Non-Life Insurance Mathematics," 2nd Edition, Springer-Verlag, Berlin, 2009. doi:10.1007/978-3-540-88233-6

[5] M. Morales, "On a Surplus Process under a Periodic Environment: A Simulation Approach," North American Actuarial Journal, Vol. 8, No. 2, 2004, pp. 76-87.

[6] Y. Lu and J. Garrido, "Doubly Periodic Non-Homogeneous Poisson Models for Hurricane Data," Statistical Methodology, Vol. 2, No. 1, 2005, pp. 17-35. doi:10.1016/j.stamet.2004.10.004

[7] J. Garrido and Y. Lu, "On Double Pariodic Non-Homogeneous Poisson Processes," Bulletin of the Association of Swiss Actuaries, Vol. 2, 2004, pp. 195-212.

[8] U. Jaroengeratikun, W. Bodhisuwan and A. Thongteeraparp, "A Statistical Analysis of Intensities Estimation on the Modeling of Non-Life Insurance Claim Counting Process," Applied Mathematics, Vol. 3, No. 1, 2012, pp. 100-106. doi:10.4236/am.2012.31016

[9] P. Mukhopadhyay, "An Introduction to Estimating Functions," Alpha Science International Ltd., Harrow, 2004.

[10] P. K. Andersen, O. Borgan, R. D. Gill and N. Keiding, "Statistical Models Based on Counting Processes," Springer-Verlag New York, Inc., New York, 1993.

[11] P. Yip, "Estimating the Number of Error in a System Using a Martingale Approach," IEEE Transactions on Reliability, Vol. 44, No. 2, 1995, pp. 322-326. doi:10.1109/24.387389

[12] J. E. R. Cid and J. A. Achcar, "Bayesian Inference for Nonhomogeneous Poisson Processed in Software Reliability Models Assuming Nonmonotonic Intensity Functions," Computational Statistics \& Data Analysis, Vol. 32, No. 2, 1999, pp. 147-159. doi:10.1016/S0167-9473(99)00028-6

[13] J. Gill, "Bayesian Methods: A Social and Behavioral Sciences Approach," Chapman and Hall/CRC, London, 2008.

[14] T. Hirata, H. Okamura and T. Dohi, "A Bayesian Inference Tool for NHPP-Based Software Reliability Assessment," In Y. H. Lee, T.-H. Kim, W.-C. Fang and D. Slezak, Eds., Lecture Notes in Computer Science, Vol. 5899, Springer, Berlin, 2009, pp. 225-236.

[15] D. P. M. Scollnik, "Actuarial Modeling with MCMC and BUGS," North American Actuarial Journal, Vol. 5, No. 2, 2001, pp. 96-124.

[16] X. Zhao, C. Yu and H. Tong, "A Bayesian Approach to Weibull Survival Model for Clinical Randomized Censoring Trial Based on MCMC Simulation," The 2nd International Conference on Bioinformatics and Biomedical Engineering, Shanghai, 16-18 May 2008, pp. 1181-1184. 